\title{
Clustering and Resilience in Cloud Video Conference Solutions
}

\author{
Siddarth Kaul
}

\begin{abstract}
The paper proposes to clearly elaborate the usefulness and necessity of clustering and Resilience in Video Conferencing with Cloud Computing it deeply focuses on key areas of how the video conferencing solutions in cloud environment are positioned with an intense resilience on failover and innovations in new technologies for reduced infrastructure usage and less down time with new features availability in cloud environment. The paper also proposes to give a new insight in the various other functions of cloud computing terminologies like Virtualization and Grid Computing in the cloud.
\end{abstract}

Index Terms-Cloud Computing; Video Conferencing; Virtualization and Grid Computing.

\section{INTRODUCTION}

Intelligent and Efficient Video Conferencing solutions is the demand of enterprise solutions with the new Cloud solutions the cloud videoconferencing means hosting a video Call on the internet as opposed to on premises server in the process Cloud Video has also become more useful nowadays the change is reshaping the business in a way of the powerful way of intelligent communication [1].The cost efficiency is one of the key parameters which is always kept in mind by developers (e.g. dial-in-Video and Dial-in-Audio Conferencing solution) these services are normally amalgamated to be software as a service (SAAS) the conferencing solutions which are usually provisioned involves a life cycle of the entire software as service solution like the development and deployment, provisioning video conferencing in cloud solutions the paper proposes to help understand how these challenges can be dealt with high efficiency deployment in real time scenarios [2].

\section{Cloud COMPuting}

Cloud computing a unique paradigm based on virtualization and complex IT resources on demand as service which need to be highly available anytime, anywhere via the Internet the cloud computing gives the modern age Information technology an efficient transformation to off premises, cluster, grid and distributed computing [3]. The video conferencing solutions delivered by cloud service provider depict various web-based user requirements as depicted below in Fig. 1 [4]. A cloud environment is typically a parallel and distributed system consisting of the collection of inter-connected virtualized

Published on September 27, 2018

S. Kaul, is with the Computer Science Engineering Department, Faculty of Engineering, Bhagwant University Ajmer, Rajasthan India (email siddarthkaul7@gmail.com). cloud involving multiple systems used for resource-based sharing between each other on time sharing basis [5]. Cloud resources are shared across the Globe without any limitation on software and hardware requirements.

Cloud computing deployments normally include the various type of clouds the Public Cloud, Private Cloud, Hybrid and the Community Cloud. Cloud computing can also be used for various applications of voice and Video Conferencing as shown in Fig. 2 [6].

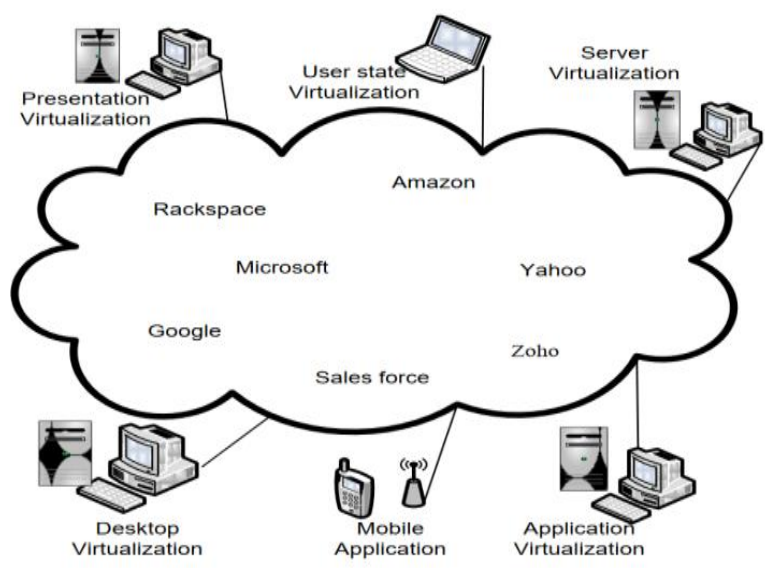

Fig. 1. Cloud Computing Solutions

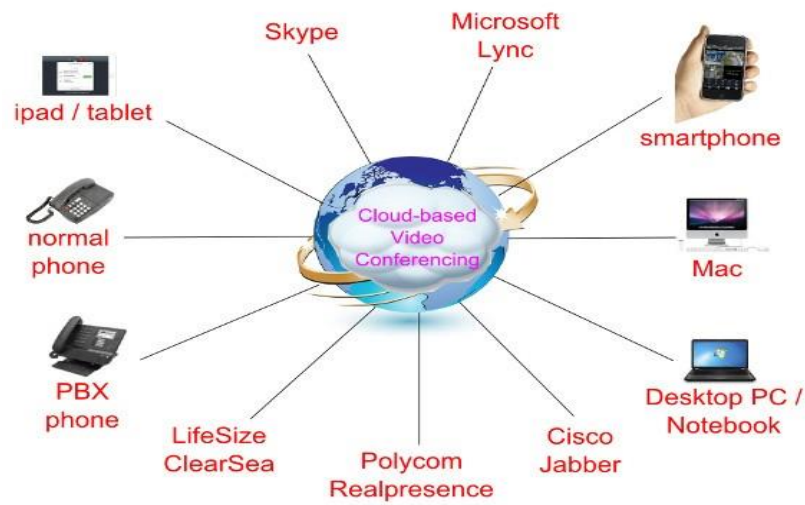

Fig. 2. Voice and Video Conferencing solutions on cloud

\section{Clunster COMPUTING}

The cluster computing can be described as the combined fusion entity of Parallel, High Performance, Distributed high availability computing. The use of cluster as shown in Fig. 3 is based on the communication between nodes and designing fast and robust low latency networks, Cluster computing also provides a wide range of platform for solving distinct parallel and distributed applications used in scientific and commercial areas nowadays cluster computing is also used for supercomputing applications such as earthquake, systemic and hurricane predictions [7]. Clusters 
can also be used in various applications involving data mining and other commercial applications such as Image rendering and network simulation. The cluster in computing is not specific to Industrial applications they are also used in high speed mobile networks such as 5G and LTE (Long term Evolution) [8].

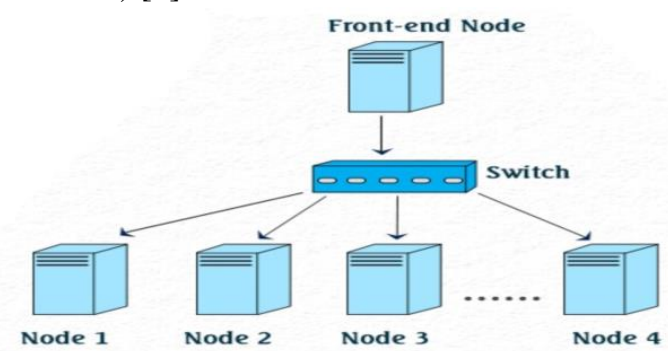

Fig. 3. Cluster in Computer Networks

\section{Cluster COMPuting IN VIDEO CONFERENCING}

The cluster computing in video conferencing as described is a combination of server networking techniques and server pool networking for building super clusters that not only support large number of users and concurrent calls but also provides excellent reliability and resilience to failures. Fig. 4 depicts a super cluster with high resilience.

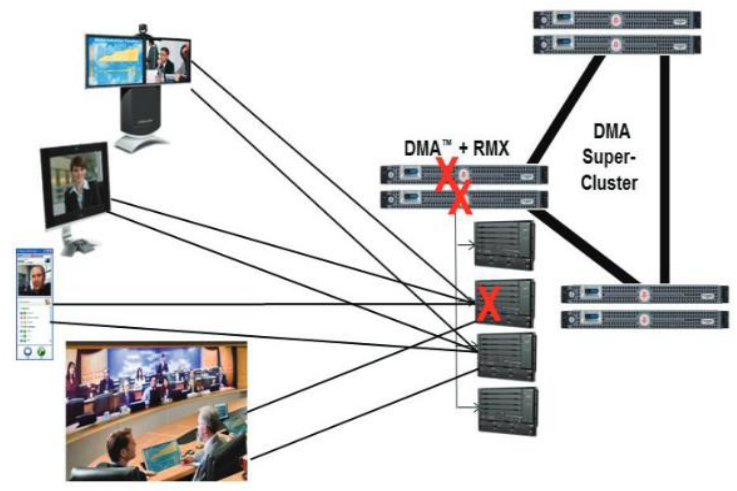

Fig. 4. Super cluster in Video Conferencing

Supercluster scenario as shown in Fig. 4 also depicts a Conference Bridge solution with conferencing server and super nodes registration in resiliency. If one node has to be taken down for maintenance, another node in super cluster takes over. If the entire super node fails, the cluster takes over that means the calls are processed by other nodes in cluster. If the new conference server fails over the conferences are moved to next server once, the calls stay up or new calls are added are connected to new conference server [9].

\section{GRID COMPUTING}

Grid computing is a unique group of networked computers which work together to act as a virtual super computer to perform various large are bulk tasks such as analyzing huge sets of data or weather modelling. High performance computers and scientific databases and scientific instruments are connected using Grid Computing,
Grid computing enables an integrated collaborative use of system that coordinates the resources to centralized control using various open standards and protocols with non-trivial quality of service parameters in engagement with Cloud computing. Grid computing in a cloud environment can be depicted as in Fig. 5 [10].

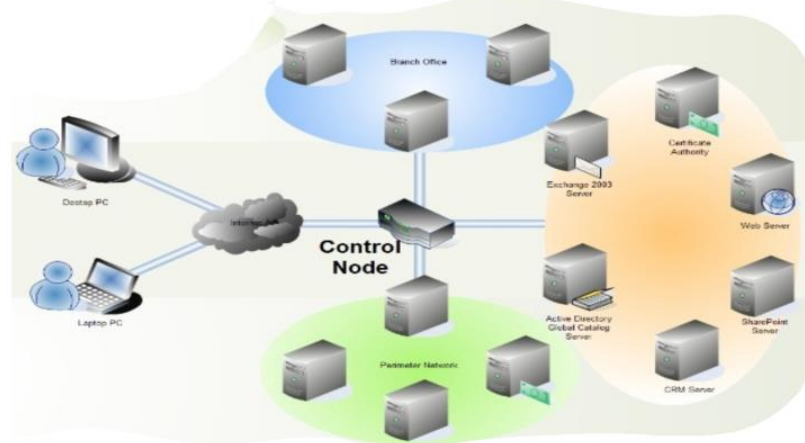

Fig. 5. Grid Computing in Cloud

\section{GRID COMPUTING IN VIDEO CONFERENCING}

Grid computing in Video Conferencing can be used in the same conceptual concept as Cloud computing however the Grid computer together Integrates various protocols like SIP (Session Initiation Protocol) and H.323 endpoints in one frame work for $\mathrm{A} / \mathrm{V}$ collaboration systems. The Fig. 6 depicts a typical Audio Video Conferencing system which uses Grid Computing cloud in with a SIP (Session Initiation Protocol) gateway, Client and H.323 gateway, Client both using a grid computing cloud which helps in high resiliency of video conferencing despite one service failing will help failover to another service [11].

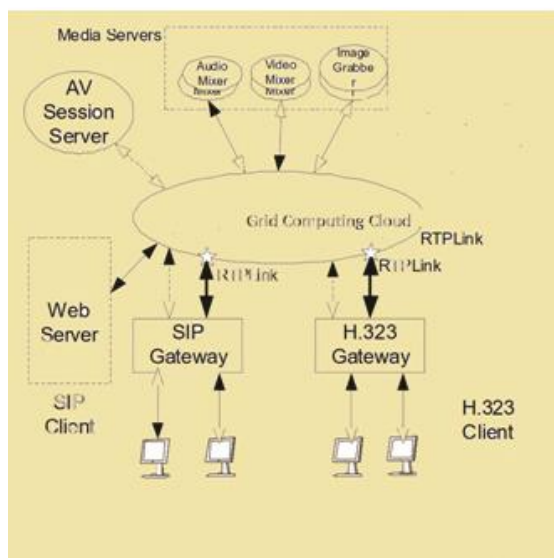

Fig. 6. Grid Computing in Video Conferencing

\section{DISCUSSION ON CLOUD, CLUSTER AND GRID COMPUTING IN VIDEO CONFERENCING}

There are several development levels which video conferencing solutions can be dealt with these parameters include ownership, type, service, pricing, virtualization etc. [12]. The overall solutions in video conferencing is distributed with the help of resources administratively located and managed through the cloud solutions with their own respective management goals and policies [13] 


\section{CONCLUSION}

The cloud computing pad grim has evolved to be a distinctive solution which has helped the further amalgamation of all other solutions to provide all services in distinct format of SAAS (Software as Service), PAAS (Platform as Service) on as the combined forefront of Cloud Computing. The paper has been used to present the relationship, Differences in cloud computing to other supported technologies in the Video Conferencing needs evolving in today complex business needs.

\section{REFERENCES}

[1] N Sadashiv and S D Kumar "Cluster and Cloud Computing: A detailed Comparison "2011 6 th conference on computer science and education, 2011 pp. 477-483.

[2] https://arxiv.org/ftp/arxiv/papers/1605/1605.00305.pdf pp. 1- 2 Introduction Cloud Platform as service for Multimedia Conferencing service provisioning.

[3] S. Zhang, X. Chen, X Huo "The comparison between cloud computing and Grid Computing" 2010 International conference on computer application and system Modelling volume 11 pp. $10-71$

[4] http://www.ijfcc.org/Vol4/361-CS306.pdf section cloud computing description.

[5] https://www.investopedia.com/terms/c/cloud-computing.asp section what is cloud computing and Breaking down cloud computing.

[6] http://www.eurekaplus.com/images/video-conferencing/cloud-basedvideo-conferencing-compatible-endpoints description and Detailed prospective.
[7] https://www.google.com/search?q=cluster+computing\&client=firefox -b-ab\&source a detailed overview section cluster computing pp. 1113.

[8] R Buyya, C.S Yeo, S Venugopal, J Broberg and I Brandic cloud computing an emerging IT platform, Vision, hype and reality for delivering computing as the 5th utility "Future Generation Computer systems, Vol 25 no 5 , no 6 pp. 551 -615.

[9] J. et al, 2002. RFC 3261 SIP: Session Initiation Protocol. IETF Request for Comments, June 2002. http://www.ietf.org/rfc/rfc3261.txt

[10] http://ecomputernotes.com/fundamental/introduction-tocomputer/grid-computing detailed dialogue.

[11] http://www.cloudbus.org/papers/FGCS-Cluster.pdf

[12] https://surface.syr.edu/cgi/viewcontent.cgi?article=1131\&context=eec $\mathrm{s}$ section A/V Collaboration architecture pp. 4-7

[13] https://surface.syr.edu/cgi/viewcontent.cgi?article=1131\&context=eec $\mathrm{s}$ section A/V Collaboration architecture pp. 1-2

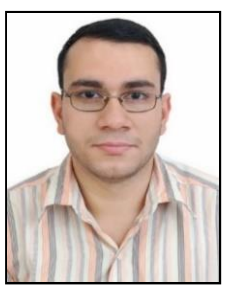

Siddarth Kaul received his Diploma in Electronics and Communication Engineering from Board of Technical Education, Delhi, India, and B.Tech degree in Electronics and Communication Engineering from GGSIP University, Delhi India in 2011, PGDCA from Sam Higgin bottom Institute of Agriculture, Technology and Sciences, Allahabad, India, MSC (IT) from Lovely Professional University, Punjab, India \& M.Tech degree in Software Engineering from Singhania University, Rajasthan, India in 2013. He is presently pursuing Ph.D. in Computer Science Engineering from Bhagwat University, Ajmer, Rajasthan, India. He is a certified CCNA (Voice) professional and he has worked with TCIL (Telecommunication Consultants India Limited) on overseas project for two years as VSAT Engineer. He has also worked on Panterra Networks as Engineer Level 3 in Software Engineering Division. He had worked as Voice Engineer with Polycom, India before Joining Atkins (SNC - LAVALIN Company). 